\title{
Violência urbana e política pública de prevenção: avaliação do Programa Fica Vivo! no estado de Minas Gerais, Brasil ${ }^{\star}$
}

\author{
Bráulio Figueiredo Alves Silva* \\ Bernardo Lanza Queiroz ${ }^{\star \star \star}$ \\ Frederico Couto Marinho ${ }^{* * *}$ \\ Fabiano Neves Alves Pereira ${ }^{\star \star \star \star \star}$ \\ Pedro Cisalpino ${ }^{\star \star \star \star \star \star}$
}

\begin{abstract}
A violência urbana no Brasil tem assumido proporções elevadas nos últimos anos. Somente em 2014, quase 60 mil pessoas foram vítimas de homicídios no Brasil, posicionando o país entre os mais violentos do mundo. 0 objetivo deste texto é apresentar os resultados da avaliação da implementação do Programa Fica Vivo! na cidade de Belo Horizonte, ao longo de 15 anos de execução. De maneira geral, pode-se dizer que o programa cumpriu sua finalidade e logrou êxito em reverter a tendência de crescimento das taxas de homicídio. A metodologia adotada para avaliar os impactos do Programa Fica Vivo! permitiu aferir os efeitos das políticas de prevenção e controle dos homicídios.
\end{abstract}

Palavras-chave: Avaliação de políticas públicas. Diferenças em diferenças. Pareamento por escore de propensão. Programa Fica Vivo! Homicídios. Belo Horizonte.

\footnotetext{
* Resultado de pesquisa do Projeto BRA/04/029 - Segurança Cidadã - Projeto Pensando a Segurança Pública. Edital de Convocação no 002/2015 - Seleção de Projetos. Ministério da Justiça / Secretaria Nacional de Segurança Publica.

** Departamento de Sociologia, Centro de Estudos de Criminalidade e Segurança Pública, Universidade Federal de Minas Gerais (UFMG), Belo Horizonte-MG, Brasil (braulio.fas@gmail.com; https://orcid.org/0000-0003-1791-9075).

*** Centro de Desenvolvimento e Planejamento Regional (Cedeplar), Universidade Federal de Minas Gerais (UFMG), Belo Horizonte-MG, Brasil (blanza@gmail.com; http://orcid.org/0000-0002-2890-1025).

**** Instituto de Geociências, Universidade Federal de Minas Gerais (UFMG), Belo Horizonte-MG, Brasil (fcfrederico9@ gmail.com; http://orcid.org/0000-0002-1405-3125)

${ }_{\star \star \star \star \star}$ Centro de Desenvolvimento e Planejamento Regional (Cedeplar), Universidade Federal de Minas Gerais (UFMG), Belo Horizonte-MG, Brasil (fnapereira@cedeplar.ufmg.br; http://orcid.org/0000-0002-0784-3233)

${ }^{\star \star \star \star \star \star}$ Centro de Desenvolvimento e Planejamento Regional (Cedeplar), Universidade Federal de Minas Gerais (UFMG), Belo Horizonte-MG, Brasil (pedrocisalpino@gmail.com; http://orcid.org/0000-0002-6954-1708).
} 


\section{Introdução}

Historicamente, o número absoluto de homicídios no Brasil tem aumentado gradativamente, colocando o país entre os mais violentos do mundo. A despeito das distintas explicações apontadas por inúmeros estudos, nos âmbitos nacional e internacional há alguns consensos acerca dessa problemática: o primeiro refere-se ao recrudescimento da violência na população masculina jovem; e o segundo consiste na evidência cada vez maior da concentração espacial da violência, particularmente a elevada incidência de homicídios em aglomerados urbanos caracterizados por uma grande vulnerabilidade social (BARATA; RIBEIRO; MORAES, 1999; BARROS; XIMENES; LIMA, 2001; CDC, 2010; BEATO FILHO, 2012).

Em decorrência disso, os moradores de lugares com essa característica social são incapazes de compartilhar os mesmos valores sociais e morais, tampouco se mobilizar para solucionar problemas comuns, como a vulnerabilidade e o envolvimento de adolescentes e jovens em atos infracionais (tráfico de drogas, violência interpessoal, posse de arma de fogo) (BERRY; KASSARDA, 1997). Tomado em conjunto, o resultado é uma limitada capacidade local em estabelecer vínculos comunitários e institucionais com fontes de recursos fundamentais para o estabelecimento da ordem social local (BERRY; KASSARDA, 1997; SAMPSON; GROVES, 1989).

Nesse contexto, alguns autores preocupados em compreender esse processo de violência urbana, concentração espacial de homicídios e grande vitimização de jovens, de maneira geral, ressaltam a importância de se considerar a esfera pública como promotora da organização social dos lugares tradicionalmente violentos, aumentando os laços sociais entre os vizinhos, criando uma rede de confiança, participação e envolvimento comunitário e, principalmente, promovendo ações que têm como objetivo a redução da violência.

Assim, é importante observar o papel do componente institucional como um estoque de recurso de organizações presentes na vizinhança (igrejas, escolas, associações de bairro, etc.) e de sua capacidade de interlocução com organizações externas à comunidade (Secretarias Municipais, governo estadual, Polícia, Ministério Público, Judiciário, etc.).

Se, por um lado, nos últimos anos é possível identificar uma série de políticas públicas voltadas para a redução da criminalidade no Brasil, por outro, pouco esforço tem sido dedicado a avaliar o impacto dessas ações (ANGRIST; PISCHKE, 2008; VOCHT et al., 2015; LÉRIDA, 2006). 0 que está subjacente a essa discussão é o fato de que a concepção e a efetiva implementação de uma política pública de controle de homicídios, que tem uma faceta multisetorial de investimentos para a comunidade local, além de contribuir para a redução a curto prazo da incidência de mortalidade por causa da violência interpessoal, promovem uma melhoria das percepções sobre sua própria comunidade.

Este texto tem dois propósitos: pretende suprir uma lacuna na produção nacional apresentando resultados da avaliação de políticas públicas de prevenção à criminalidade e violência; e busca avaliar a implementação do Programa Fica Vivo! na cidade de Belo 
Horizonte ao longo de 15 anos de execução, no que se refere à redução de homicídios nos locais onde ocorreram as ações do programa.

\section{O programa e o cenário de estudo}

O Fica Vivo! é um programa do governo do Estado de Minas Gerais que tem como objetivo reduzir a incidência de homicídios dolosos, mediante ações de prevenção e repressão, em áreas com altos níveis de criminalidade violenta, a partir de ações integradas dos governos federal, estadual e municipal, do Ministério Público, do Poder Judiciário, bem como das organizações não governamentais e da sociedade civil. Inspirado em experiências bem-sucedidas empreendidas em cidades como Boston e Bogotá, o Fica Vivo! é considerado um programa inovador no que se refere à concepção e implementação de políticas de segurança pública no Brasil.

O programa foi iniciado em Belo Horizonte, em 2002, num contexto em que os homicídios começavam a atingir patamares críticos. Entre 2004 e 2014, o programa ultrapassou os limites da capital mineira e expandiu-se para outros 12 municípios do estado de Minas Gerais. Ao todo, foram implementados 31 Núcleos de Prevenção à Criminalidade (NPC) de Base Local, que são os equipamentos instalados exatamente nos territórios atendidos para o desenvolvimento do Programa Fica Vivo!

\section{Metodologia}

Para analisar o comportamento da incidência de homicídios nas áreas onde o programa foi implementado, a fim e avaliar seu impacto com relação a essa modalidade de crime, foi preciso adotar a seguinte estratégia de análise: num primeiro momento, por meio da utilização de uma metodologia conhecida por escore de pareamento, foram selecionadas áreas similares àquelas onde o Fica Vivo! foi implantado no que diz respeito a indicadores sociodemográficos (SIGNORINI; QUEIROZ, 2011). No momento seguinte, implementou-se o método conhecido como diferenças em diferenças, em que as unidades de análises obtidas na etapa anterior foram divididas em quatro grupos: o de controle antes da mudança; o de controle depois da mudança; o de tratamento antes da mudança; e o grupo de tratamento depois da mudança.

\section{Pareamento por escore de propensão}

O objetivo desse recurso metodológico relativo ao pareamento é encontrar um grupo de comparação ideal em relação ao de tratamento, a partir de uma amostra de não participantes. A relação de proximidade entre os grupos é medida em termos das características observáveis (LÉRIDA, 2006; SIGNORINI; QUEIROZ, 2011).

Nesse estudo, usamos o método de pareamento de Kernel, em que todas as unidades tratadas (áreas onde foi implementado o Programa Fica Vivo!) são pareadas com uma média 
ponderada de todas as unidades do grupo de controle (áreas similares sem o Programa Fica Vivo!). 0 estimador de Kernel é dado por:

$\tau^{K}=\frac{1}{N^{T}} \sum_{i \in T}\left\{Y_{i}^{T}-\frac{\sum_{j \in C} Y_{j}^{C} G\left(\frac{p_{i}-p_{i}}{h_{n}}\right)}{\sum_{k \in C} G\left(\frac{p_{k}-p_{i}}{h_{n}}\right)}\right\}$

Onde: G(.) é a função de Kernel e $h n$ é um parâmetro da largura da banda (bandwidth). Sob condições padronizadas, a largura da banda e o Kernel são estimadores consistentes da resposta contrafactual $Y_{i 0}$.

$\frac{\sum_{j \in C} Y_{j}^{C} G\left(\frac{p_{i}-p_{i}}{h_{n}}\right)}{\sum_{k \in C} G\left(\frac{p_{k}-p_{i}}{h_{n}}\right)}$

De forma simplificada, apresentam-se, a seguir, os critérios utilizados para seleção do grupo de controle e também os resultados da aplicação do propesity score matching.

Primeiramente, definimos quatro variáveis de controle para a comparação dos grupos:

- proporção de domicílios com coleta de lixo (Prop_dom_coleta);

- proporção de responsáveis pelo domicílio com renda de até um salário mínimo (Prop_PRR1SM);

- proporção de responsáveis pelos domicílios de cor/raça branca (Prop_BRA);

- proporção de homens de 15 a 29 anos nos setores censitários (Prop_H1529).

Depois disso, decidiu-se filtrar a busca a partir de dois parâmetros:

- serem idênticas às áreas de tratamento, tendo como referências as quatro variáveis acima;

- diferenciar em média no máximo 5\% em cada uma das variáveis acima.

Após a realização do procedimento foram selecionados 510 setores censitários sem a presença do programa que melhor se compatibilizaram com grupo de tratamento segundo os critérios definidos.

\section{Diferenças em diferenças}

Esse método pode ser representado pela equação de regressão linear apresentada a seguir. Nesse estudo, considerou-se utilizar um modelo enxuto sem o controle de outras variáveis externas, que é representado pela seguinte equação (ANGRIST; PISCHKE, 2008; PEREIRA; QUEIROZ, 2016).

$\hat{Y}=\alpha+\beta_{1} T_{i}+\beta_{2} t_{i}+\beta_{3}\left(T_{i}^{*} t_{i}\right)+\varepsilon_{i}$

Onde: $\hat{Y}$ representa a variável estudada; $\alpha$ é o valor da variável estudada quando se analisa o grupo de controle antes da política; $\beta_{1}$ refere-se ao impacto do grupo de tratamento na variável estudada; $\beta_{2}$ é o impacto do segundo período sobre a variável estudada; $\beta_{3}$ representa o impacto pós-evento do grupo de tratamento, em relação ao grupo de controle, sobre a variável estudada; e $\varepsilon_{i}$ corresponde ao erro aleatório não observado. 


\section{Resultados}

Diante dos dois grupos utilizados para comparação, o primeiro passo foi traçar a tendência de homicídios observados nessas duas áreas ao longo dos anos analisados, por meio da média anual nos grupos e da proporção de homicídios que cada grupo tem com relação ao total verificado nas duas áreas. É possível notar que, ao longo do tempo, a proporção de homicídios ocorridos nas áreas com a presença do programa apresenta tendência de queda, principalmente a partir de 2004 (Gráfico 1).

\section{GRÁFICO 1}

Proporção de homicídios nas áreas com e sem o Programa Fica Vivo! Belo Horizonte - 1998-2015

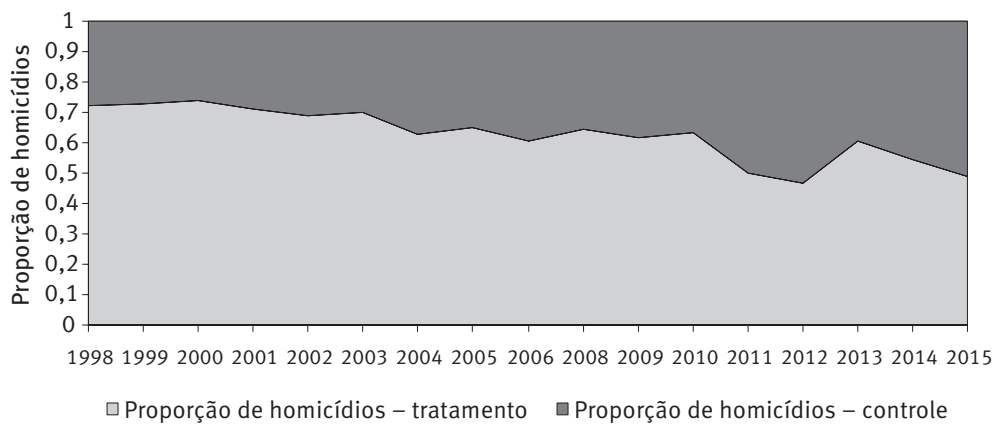

Fonte: Observatório de Segurança Pública Cidadã / Secretaria de Estado de Segurança Pública / Governo de Minas Gerais. Elaboração dos autores.

No entanto, embora essa parte das análises permita uma comparação entre grupos similares em termos sociodemográficos, é preciso aprofundar a investigação a fim de encontrar evidências estatisticamente significativas para certificar-se de tal afirmação. Nesse sentido, optou-se por implementar outra metodologia capaz de dar subsídios sobre a real efetividade do programa nas áreas em que foi implementado. 0 recurso utilizado, para tanto, é conhecido como método das diferenças em diferenças, em que as unidades de análises foram divididas em quatro grupos: o de controle antes da mudança; o de controle depois da mudança; o de tratamento antes da mudança; e o de tratamento depois da mudança.

Como se pode verificar, a partir dos resultados apresentados na Tabela 1, a incidência média de homicídios no grupo de controle passou de 0,163 para 0,217 se comparados os períodos antes e depois de 2005, quando de fato iniciou-se o processo de implementação e expansão do programa em Belo Horizonte. Para o grupo de tratamento, por outro lado, observa-se o oposto, com redução de quase $20 \%$ da incidência média no mesmo período de comparação. É possível constatar também que as diferenças entre os grupos de tratamento e controle antes e depois da implantação do programa são estatisticamente válidas. 
TABELA 1

Método de diferenças em diferenças antes e depois de 2005 para a média de homicídios por setor censitário com e sem o Programa Fica Vivo! - Belo Horizonte

\begin{tabular}{lcc|lcc}
\hline \multicolumn{1}{c}{ Grupo controle } & $\begin{array}{c}\text { Antes de } \\
\mathbf{2 0 0 5}\end{array}$ & $\begin{array}{c}\text { Depois } \\
\text { de 2005 }\end{array}$ & Grupo de tratamento & $\begin{array}{c}\text { Antes de } \\
\mathbf{2 0 0 5}\end{array}$ & $\begin{array}{c}\text { Depois } \\
\text { de 2005 }\end{array}$ \\
\hline Média de homicídios por setor & $\mathbf{0 , 1 6 3}$ & $\mathbf{0 , 2 1 7}$ & Média de homicídios por setor & 0,327 & 0,266 \\
Desvio padrão & $\mathbf{0 , 2 7 7}$ & $\mathbf{0 , 2 5 7}$ & Desvio padrão & 0,436 & 0,296 \\
Número de observações & 3.570 & $\mathbf{4} .590$ & Número de observações & 3.962 & 5.094 \\
Estatística IC & 0,009 & $\mathbf{0 , 0 0 7}$ & Estatística IC & 0,014 & 0,008 \\
Limite inferior IC & $\mathbf{0 , 1 5 4}$ & $\mathbf{0 , 2 0 9}$ & Limite inferior IC & 0,313 & 0,258 \\
Limite superior IC & $\mathbf{0 , 1 7 2}$ & $\mathbf{0 , 2 2 4}$ & Limite superior IC & 0,341 & 0,274 \\
\hline
\end{tabular}

Fonte: Observatório de Segurança Pública Cidadã / Secretaria de Estado de Segurança Pública / Governo de Minas Gerais. Tabulação dos autores.

Os resultados também permitem dizer que, em média, a implantação do programa reduz em 0,115 a média de homicídios, por ano, nas áreas em que o programa atua. Os impactos do Programa Fica Vivo! são mais nítidos quando observamos a diferença entre a tendência com base na média de variação de homicídios observada antes da implementação do programa e a tendência que incorpora a variação dos homicídios após o programa. Assim, foi possível projetar o número médio de homicídios que deixaram de ocorrer nas áreas onde o programa atua devido à sua implantação. Realizou-se essa estimativa por meio da multiplicação do impacto médio pelo número total de setores censitários onde o programa atua.

Os resultados indicam que, por ano, o programa pode ter evitado em média a ocorrência de 65 homicídios, o que permite fazer uma estimativa de 650 mortes evitadas ao longo dos dez anos de funcionamento do programa. Isso corresponde a $43 \%$ do total de homicídios observados nas áreas onde o programa atua entre 2005 e 2015. Equivale também a 14\% do total de homicídios ocorridos em Belo Horizonte nesse mesmo período. 0 resultado mais surpreendente da estimativa produzida a partir dessa análise demonstra que, no período estudado, foi evitado um número de homicídios superior ao total registrado em toda a cidade de Belo Horizonte em 2015, ano em que ocorreram 582 mortes. ${ }^{1}$ Isso significa que, em dez anos de funcionamento, o Programa Fica Vivo! evitou, nas áreas onde foi implementado, o equivalente a mais de um ano de mortes por homicídio em toda a cidade de Belo Horizonte.

TABELA 2

Número médio de homicídios evitados por ano nas áreas onde o Programa Fica Vivo! foi implementado Belo Horizonte

\begin{tabular}{ll}
\hline \multicolumn{1}{c}{ Indicadores } & Dados \\
\hline Redução média por área & $-0,115$ \\
№ de áreas com o programa & 5.566 \\
Número médio de homicídios evitados por ano & 65,09 \\
\hline
\end{tabular}

Fonte: Observatório de Segurança Pública Cidadã / Secretaria de Estado de Segurança Pública / Governo de Minas Gerais. Tabulação dos autores.

\footnotetext{
${ }^{1}$ Dados disponiveis em: 〈http://www.numeros.mg.gov.br/QvAJAXZfc/opendoc.htm?document=MapaResultados.qvw\&ho st=QVS\%40vm13532\&anonymous=true\&Sheet=SHHomicidios $>$. Acesso em: 11 abr. 2018.
} 


\section{Conclusão}

0 recrudescimento da violência urbana no Brasil nos últimos anos, particularmente aquela decorrente dos homicídios, tem pautado a agenda dos governos em diferentes níveis, principalmente no que tange as políticas públicas de prevenção. Em Minas Gerais, a implementação do Programa Fica Vivo! focou em ações de prevenção e repressão, em áreas com altos níveis de criminalidade violenta, integrando esforços dos governos federal, estadual e municipal, do Ministério Público, do Poder Judiciário, bem como das organizações não governamentais e da sociedade civil. 0 projeto foi institucionalizado em 2003 e passou por um processo de expansão entre 2004 e 2014.

De maneira geral, pode-se dizer que o programa cumpriu seu respectivo objetivo e logrou êxito em reverter a tendência de crescimento das taxas de homicídio. 0 efeito da implantação do programa, considerando constante todos os outros fatores, ao longo de dez anos, foram as 650 vidas poupadas apenas na capital mineira que poderiam ter sido “ceifadas" em decorrência dos homicídios.

Diante desses resultados, é possível afirmar que, embora muitos desafios ainda persistam no âmbito da implementação de políticas públicas dessa envergadura no Brasil, a metodologia adotada para avaliar os impactos do Programa Fica Vivo! permitiu aferir os efeitos das políticas de prevenção e controle dos homicídios.

\section{Referências}

ANGRIST, J. D.; PISCHKE, J. S. Mostly harmless econometrics: an empiricist's companion. Princeton, NJ: Princeton University Press, 2008.

BARATA, R. B.; RIBEIRO, M. A. S.; MORAES, J. C. Tendência temporal da mortalidade por homicídios na cidade de São Paulo Brasil: 1979-1994. Cadernos de Saúde Pública. v. 15, n. 4, p. 711-718, 1999.

BARROS, M. D. A.; XIMENES, R.; LIMA, M. L. C. Mortalidade por causas externas em crianças e adolescentes: tendências de 1979 a 1995. Revista de Saúde Pública, v. 35, n. 2, p. 142-149, 2001.

BEATO FILHO, C. C. Crime e cidades. Belo Horizonte: Editora UFMG, 2012.

BERRY, B. J. L.; KASSARDA, J. D. Contemporany urban ecology. New York: Macmillan, 1997.

CDC - CENTER FOR DISEASE CONTROL AND PREVENTION. Homicide trends and characteristics Brazil, 1980-2002. 2010. Disponivel em: 〈http://www.cdc.gov/mmwr/preview/mmwrhtml/ mm5308a1.htm>.

LÉRIDA, J. L. de. The impact of exogenous variation in family size on women's labor force participation. In: CONFERENCE OF THE EUROPEAN SOCIETY FOR POPULATION ECONOMICS, 20, 2006, Verona. Anais... Verona: ESPE, 2006.

PEREIRA, F. N.; QUEIROZ, B. L. Diferenciais de mortalidade jovem no Brasil: a importância dos fatores socioeconômicos dos domicílios e das condições de vida nos municípios e estados brasileiros. Cadernos de Saúde Pública, v. 32, n. 9, set. 2016.

SAMPSON, R. J.; GROVES, W. B. Community structure and crime: testing social disorganization theory. American Journal of Sociology, v. 94, n. 4, p. 774-802, 1989. 
SIGNORINI, B.; QUEIROZ, B. The impact of Bolsa Família Program on beneficiaries fertility. Belo Horizonte: Centro de Desenvolvimento e Planejamento Regional, Universidade Federal de Minas Gerais (Cedeplar/UFMG), 2011 (Texto para Discussão, n. 439).

VOCHT, F.; CAMPBELL, R.; BRENNAN, A. C.; MOONEY, J.; ANGUS, C. H. M. Propensity score matching for selection of local areas as controls for evaluation of effects of alcohol policies in case series and quasi case-control designs. Public Health, v. 10, n. 1016, 2015.

\section{Sobre os autores}

Bráulio Figueiredo Alves Silva é doutor e mestre em Sociologia pela Universidade Federal de Minas Gerais (UFMG). Professor adjunto do departamento de Sociologia da UFMG, pesquisador do Centro de Estudos de Criminalidade e Segurança Pública e do Centro de Pesquisas Quantitativas em Ciências Sociais da UFMG. Membro pesquisador do INCT Espaço Urbano e Gestão da Segurança Pública. Bolsista de Produtividade CNPq (categoria 2).

Bernardo Lanza Queiroz é Ph.D em Demografia pela Universidade da California, Berkeley.

Frederico Couto Marinho é professor adjunto do Instituto de Geociências na Universidade Federal de Minas Gerais (UFMG).

Fabiano Neves Alves Pereira é doutorando e mestre em Demografia pela Universidade Federal de Minas Gerais (UFMG).

Pedro Cisalpino é doutorando em Demografia no Centro de Desenvolvimento e Planejamento Regional (Cedeplar), Universidade Federal de Minas Gerais (UFMG).

\section{Endereço para correspondência}

\section{Bráulio Figueiredo Alves Silva}

Centro de Estudos de Criminalidade e Segurança Pública (Crisp/UFMG)

Av. Presidente Antônio Carlos, 6627, Unidade Administrativa III, Pampulha

31270-901 - Belo Horizonte-MG, Brasil

\section{Bernardo Lanza Queiroz}

Faculdade de Ciências Econômicas (Face/UFMG) - campus Pampulha

Av. Presidente Antônio Carlos, 6627, Pampulha

31270-901 - Belo Horizonte-MG, Brasil

Frederico Couto Marinho

Rua Desembargador Paula Mota, 888, apto 301

31310340 - Belo Horizonte-MG, Brasil

\section{Fabiano Neves Alves Pereira}

Rua Bahia, casa 2, São Benedito - Santa Luzia

33125-400 - Belo Horizonte-MG, Brasil

Pedro Cisalpino

Rua Tenente Anastácio de Moura, 1057/201, Santa Efigênia

30240-390 - Belo Horizonte-MG, Brasil 


\begin{abstract}
Urban violence and public prevention policy: assessment of Program "Fica Vivo" (is alive), in the State of Minas Gerais, Brazil

Urban violence in Brazil has reached high proportions in recent years. Only in 2014, almost 60 thousand people were victims of homicide in Brazil, positioning the country among the most violent in the world. The goal of this research note is to present the evaluation of the implementation of the Fica Vivo Program in the city of Belo Horizonte over 15 years of execution. In general, it can be said that the program has fulfilled its objective and has succeeded in reversing the growth trend of homicide rates. The methodology adopted to evaluate the impacts of the Fica Vivo Program allowed to assess the effects of policies of prevention and control of homicide.
\end{abstract}

Keywords: Public policies evaluation. Difference in difference. Propensity score matching. Stay Alive Program. Homicides. Belo Horizonte.

\title{
Resumen
}

Violencia urbana y política pública de prevención: evaluación del Programa "Fica Vivo" en el Estado de Minas Gerais, Brasil

La violencia urbana en Brasil ha asumido proporciones elevadas en los últimos años. Solo en 2014, casi sesenta mil personas fueron víctimas de homicidios en Brasil, lo que posicionó al país entre los más violentos del mundo. El objetivo de este artículo es mostrar el resultado de la evaluación de la implementación del programa Fica Vivo en la ciudad de Belo Horizonte a lo largo de sus 15 años de ejecución. En general, se puede decir que el programa ha cumplido su objetivo y que fue exitoso al revertir la tendencia de crecimiento de las tasas de homicidio. La metodología adoptada para evaluar los impactos del programa Fica Vivo permitió evaluar los efectos de las políticas de prevención y control de los homicidios.

Palabras clave: Evaluación de políticas públicas. Diferencias en diferencias. Pareamiento por escala de propensión. Programa Fica Vivo. Homicidios. Belo Horizonte.

Recebido para publicação em 18/05/2018

Aceito para publicação em 23/10/2018 